\section{SP0092 REGULATION OF NEUTROPHIL APOPTOSIS DURING ACUTE INFLAMMATION - A ROLE FOR THE BCL-2 FAMILY OF APOPTOSIS-ASSOCIATED GENES}

B Walzog, P Weinmann, P Gaehtgens. Physiology, Freie Universität Berlin, Berlin, Germany

\subsection{6/annrheumdis-2001.38}

Introduction The life-span of circulating polymorphonuclear neutrophils $(\mathrm{PMN})$ is relatively short when compared to other leukocytes but it can be further shortened by accelerating apoptosis. This is thought to be critical for the control of acute inflammation since apoptosis allows the "silent" elimination of emigrated PMN and thereby prevents uncontrolled tissue damage. This study was undertaken to elucidate the mechanisms which determine the life-span of emigrated PMN during acute inflammation.

Methods Apoptosis of isolated human PMN was investigated by (1) analysis of DNA content, (2) detection of DNA degradation, (3) analysis of nuclear morphology, and (4) measurement of CD16 expression on the cell surface. Gene expression was analysed by semi-quantitative RT-PCR and western blotting technique. Intraperitoneal thioglycollate injection in mice was used as an in vivo model for acute inflammation.

Results Leukocyte adhesion molecules of the $\beta 2$ integrin family (CD11/CD18) which are critically involved in the recruitment of PMN to sites of inflammation were found to potentiate TNF $\alpha$ induced apoptosis of human PMN within $4 \mathrm{~h}$ and $8 \mathrm{~h}$ after stimulation. The effect required aggregation of the $\beta 2$ integrin Mac1 (CD11b/CD18) and was independent of Fc receptors. An enhancement of apoptosis was also observed after migration of PMN through an endothelial cell monolayer which stained positively for ICAM-1, the ligand for the $\beta 2$ integrins LFA-1 (CD11a/CD18) and Mac-1 (CD11b/CD18) suggesting that PMN emigration promotes apoptosis. This was confirmed using an in vivo model of acute inflammation in mice. Moreover, inflammatory, i.e. emigrated PMN showed a differential expression of apoptosis-associated genes of the bcl-2 family when compared to circulating PMN: Whereas the pro-apoptotic factor bax-alpha was up-regulated, the expression of the anti-apoptotic bcl-XL was down-regulated in emigrated PMN. Since the bcl-2 family plays an important role in the regulation of apoptosis, this shift of balance between anti- and pro-apoptotic factors may be responsible for the enhancement of apoptosis in inflammatory PMN.

Conclusions The present study suggests a physiologically relevant mechanism for the control of acute inflammation by promoting the turnover of previously emigrated PMN. This mechanism which involves the bcl-2 family of apoptosis-associated genes may be critical to subside local inflammatory processes in vivo.

\section{REFERENCES}

1 Walzog B, Jeblonski F, Zakrzewicz A, Gaehtgens P. FASEB J. 1997;11:1177-86

2 Weinmann P, Gaehtgens P, Walzog B. Blood 1999;93:3106- 15

3 Walzog B, Gaehtgens P. News Physiol Sci. 2000;15:107-13

\section{SP0093 JUNCTIONAL ADHESION MOLECULE-2 (JAM-2) IS LOCALISED AT INTERCELLULAR JUNCTIONS AND PLAYS A ROLE IN LYMPHOCYTE TRANSMIGRATION}

B Imhof, CA Johnson-Léger, M Aurrand-Lions, C Wong. Of Pathology, Centre Médical Universitaire, Geneva 4, Switzerland
We identified a novel Junctional Adhesion Molecule by a selective RNA display method. The protein was named JAM-2 based on its 30-40\% homology to JAM and VE-JAM. The three JAMs define a novel subset of immunoglobulin superfamily molecules which will be referred to as JAM-1 (JAM), JAM-2 and JAM-3 (VE-JAM). JAM-2 is highly expressed by HEVs and lymphatic endothelial cells in lymphoid organs, suggesting that it may play a role in lymphocyte recirculation. The role of JAM-2 in interendothelial junctions has been addressed using endothelial cells over-expressing JAM-2 protein. These cells have an increased ability to sustain lymphocyte transmigration in agreement with the pattern of expression found in vivo. Transfected cells are also more permeable to FITC-dextran. Furthermore, an antibody directed against the extracellular domain of JAM-2 is able to partially block the transmigration. Our findings suggest that JAM-2 may facilitate lymphocyte extravasation across HEVs by rendering the inter-endothelial junctions more leaky. The role of JAM-2 in inflammatory processes will be discussed.

\section{REFERENCE}

1 Aurrand-Lions M, Duncan L, Ballestrem C, Imhof BA. JAM-2, a novel immunoglobulin superfamily molecule, expressed by endothelial and lymphatic cells. J Biol Chem. 2001;276:2733-41

\section{SP0094 RHEUMATOLOGY DEVELOPMENT: HOW TO REACH THE EQUALLY AND HIGH STANDARD EDUCATED AND TRAINED HEALTH PROFESSIONAL THROUGHOUT EUROPE}

AD Woolf. Department of Rheumatology, Royal Cornwall Hospital, Truro, UK

\subsection{6/annrheumdis-2001.40}

The health-related outcome for all with musculoskeletal conditions (MSC) in Europe should be equal which requires similarities in their care. This is dependent on financial and human resources, a key one being the competency of the health professional which will be determined by their training and CME. The integrated multidisciplinary team need suitable training which must be harmonised throughout Europe to ensure the outcome of care is similar for all. Learning objectives must be established for all the professional groups relevant to their contribution to the management of MSC which may vary between countries. From this a curriculum can be developed. Different methods of teaching may be used but the tendency is towards problem-based learning integrating basic with clinical knowledge and linking all those involved in the management of similar problems. The assessment should be based on the curriculum. Standards for rheumatology have been developed in Europe for undergraduates, specialist training and CME. Similar standards need to be developed for other health professionals in rheumatology. These must reflect the educational needs which will depend on their roles in clinical practice in different countries. The ultimate measure of the quality of training of all HPs in rheumatology is the clinical outcomes achieved within the constraints of financial resources and priority given to their care.

\section{SP0095 WHAT NEW SKILLS SHOULD A RHEUMATOLOGIST ACQUIRE?}

D Bernard. Rheumatology, Hopital Roger Salengro, LILLE Cedex, France 
The first objective of the rheumatologist will be to keep the natural skills: diagnosis and management of diseases and of painful and functional disorders of the musculo-skeletal apparatus. The core curriculum of UEMS for Continuing Medical Education and Professional Development could be a good objective: a thorough theoretical knowledge must be maintained of anatomy, biochemistry, physiology, biomechanics, pathophysiology of pain, and cellular and molecular biology and genetics relevant to the rheumatic diseases. "The general attitude should be maintaining clinical skills by regular contact with people with musculo-skeletal conditions, the indications and interpretation of imaging technique such as conventional X-rays, CT scans, ultrasonography and radioisotopes techniques in the diagnosis, assessment ans monitoring of the rheumatic diseases":

-Degenerative disease of the joint and spine.

-Collagen vascular diseases (which must be considered as "rheumatology with systemic disorders" and not "internal diseases with rheumatological symptomes").

-Bone disease and calcium metabolism disorders, tumours.

-Inflammatory and metabolic arthritis.

Soft tissues (tendons and muscle) and nervous disorders.

-Spine diseases and particularly low-back pain.

In that sense the rheumatologist must keep its place of orchestra director, to maintain close co-operation with profession allied in the nmanagement of rheumatic disorders.

Through these goals, rheumatologist should stay competent to indicate, interpretate and manage explorations: imaging, to avoid the gap between radiologists and rheumatologists and let open the dialogue between practicians, but also bone measurement techniques. We would emphasise the necessary use of ultrasound in clinical practice. This imaging technique has to become a clinical tool, with the economic difficulties of the cost of the equipment.

Rheumatologist must stay performant in technics such as needle aspiration, bone or synovial biopsy, joint injection...at the least to be able to discuss indications.

In therapy, new biological development such as immunotherapy or molecular biology will be a major field of interest to avoid gap between clinical practice and biological tools, without neglecting more classical therapies such as rehabilitation.

The irruption of biomaterials and the development of percutaneous injections will change our way of therapy in a next future, sooner as we could expect, if we take into account the speed of development of biomaterial fabrication in industry project: artificial cartilage, bone replacement...

The rapid growth and ageing of the population will emphasise the necessary development of chronic disease coping and prevention and the sensibilisation of economic and politic deciders (which is one of the goal of Joint and Bone decade. This means that the rheumatologist has to develop the maintaining quality of life, and not only the function. In that sense, development psychological interventions, prevention of pain and depression would be of benefit for rheumatologist. We have to prove that illness considered as benign are important for ageing population in terms of quality of life, but also economic expanses and prevention of severe complications.

Health economics will be a real problem. What is the real benefit of medical or surgical interventions: economic benefit of modyfying drugs such as methotrexate in RA, bisphosponate in osteoporosis, slow acting drug in osteoarthritis? The answer to these questions are crucial. The practical financial costs of treatment vis a vis the cost of surgical interventions are critical to understanding the necessities of prevention and treatment.
Epidemiologic and economic studies are important to develop in the next future.

Patients information will be another challenge in term of access to care: what will be the consequences of the non access to appropriate in term of medical responsibility? What are the good answers to people when we cannot give them appropriate care for economic reasons? What are the controls in term of experimental therapy or surgery or new medical developments? All these questions have to be compared to the growing knowledge of the patients with the quantity of informations obtained through the medias and internet, the best but also the worst.

\section{Biologics in practice - Saturday 16 June, 10.00-11.45/Congress Hall}

\section{SP0097 MACROMOLECULES WITH ROLES IN CARTILAGE AND BONE HOMEOSTASIS}

D Heinegård. Cell and Molecular Biology, Lund University, Lund, Sweden

10.1136/annrheumdis-2001.42

The extracellular matrix of skeletal tissues contains molecules with key roles in tissue homeostasis. Particularly important are those that are part of the collagen network and maintains its integrity. Examples are members of the thrombospondin family, in cartilage particularly COMP, and members of the family of extracellular leucine rich repeat (LRR) proteins.

These proteins have key roles in the assembly of the collagen II fibrillar network and have the capacity to crosslink neighbouring fibrils by containing at least two functional domains, one binding to one fibril and the other binding to another fibril. An example is decorin showing an interaction site of the core proteins on one hand, while another being the dermatan sulfate side chain. Similarly fibromodulin contains structures with potential for interactions with different collagen fibrillar structures.

Current data indicate that an early event in osteoarthritis is the fragmentation of the binding proteins to separate the functional domains. This will result in an inability of the collagen network to prevent swelling of the tissue and maintain functionality.

The alterations elicit a response where molecules that have roles in catalysing collagen fibril assembly are produced. Such regulating proteins include COMP. This modular protein contains five identical subunits held together close to their C-terminus and having a $\mathrm{C}$-terminal end globular domain that promotes tight binding to collagen. COMP appears to bring together the collagen monomers and catalyse the formation of early fibrils in a chaperon like mode. It is also present, apparently crosslinking completed fibrils by simultaneously binding several collagen IX molecules that are covalently bound on the surface of the collagen II/XI fibres.

Other matrix constituents like PRELP and chondroadherin bind to cell surface molecules, apparently involved in signalling to the cells. These two molecules also bind to collagens I and II.

Cell binding proteins have key roles in bone turnover. Examples are osteopontin, a protein binding the $\alpha v \beta 3$ as well as the $\alpha 4 \beta 1$ integrin. The protein is produced by the osteoblast and binds to the bone mineralized surface. It then appears to have a central role in recruiting and promoting the development of osteoclast precursors to a fully functional cell. Osteopontin is 